\title{
Teorías feministas en el aula: una experiencia de formación e investigación con estudiantes de Ciencias de la Educación
}

\author{
Weimar Giovanni Iño Daza ${ }^{1}$ \\ ${ }^{1}$ Universidad Mayor de San Andrés. Facultad de Humanidades y Ciencias de la Educación. Avenida Villazón, 1995, Plaza del \\ Bicentenario, Monoblock Central, Zona Central. Ciudad de La Paz - Bolivia. \\ Autor para correspondencia/Author for correspondence: willkaweimar13@hotmail.com
}

RESUMEN: El presente trabajo tiene como propósito describir y presentar una experiencia de formación e investigación en teorías feministas con estudiantes de las asignaturas: Sociología General y Sociología de la Educación de la carrera Ciencias de la Educación. Por un lado, se puntualiza un breve panorama de las principales teorías feministas y su relación con la educación, en la que se hace énfasis en los feminismos ecológico y comunitario. Por otro, se desarrolla la experiencia que tiene dos aspectos, uno cognitivo que responde al aprendizaje de teorías feministas, y el segundo, la realización de una investigación coparticipativa en la que se empleó el método biográfico para recuperar testimonios, experiencias y vivencias de mujeres indígenas latinoamericanas en sus luchas por los territorios y por la naturaleza. Los resultados principales se refieren a que en el aula universitaria se puede generar espacios de discusión y diálogo de las teorías feministas y la realización de investigaciones, lo que evidencia que la educación formal desde una pedagogía comunitaria feminista pueden ser un espacio de transformación y deconstrucción de los sistemas patriarcal y neocolonial. Una de las consideraciones finales que se puede mencionar es que en las aulas se constituyen en espacios de concientización en los que se puede generar el empoderamiento y ruptura de los círculos de opresión y del saber patriarcal.

Palabras clave: Teorías Feministas y Educación, Feminismo Ecológico, Feminismo Comunitario, Método Biográfico, Pedagogía Comunitaria Feminista, Mujeres Indígenas Latinoamericanas. 


\title{
Feminist theories in the classroom: a training and research experience with students of Educational Sciences
}

\begin{abstract}
The purpose of this paper is to describe and present an experience of formation and research in feminist theories with students of the subjects: General Sociology and Sociology of Education in the Sciences of Education. On the one hand, a brief overview of the main feminist theories and their relation to education is emphasized, in which emphasis is placed on ecological and community feminisms. On the other hand, the experience is developed that has two aspects, a cognitive one that responds to the learning of feminist theories, and the second, the realization of a co-participative research in which the biographical method was used to recover testimonies, experiences and experiences of Latin American indigenous women in their struggles for territories and for nature. The main results refer to the fact that in the university classroom can be created spaces for discussion and dialogue of feminist theories and the realization of research, which shows that formal education from a feminist community pedagogy can be a space for transformation and deconstruction of the patriarchal and neocolonial systems. One of the final considerations that can be mentioned is that in the classrooms they constitute spaces of awareness in which the empowerment and rupture of the oppression and patriarchal knowledge circles can be generated.
\end{abstract}

Keywords: Feminist Theories and Education, Ecological Feminism, Community Feminism, Biographical Method, Feminist Community Pedagogy, Indigenous Latin American Women. 


\section{Teorias feministas em sala de aula: uma experiência de formação e pesquisa com alunos de Ciências da Educação}

RESUMO. Este trabalho tem como objetivo descrever e apresentar uma experiência de formação e investigação em teorias feministas com os alunos os temas: Sociologia Geral e Sociologia da Educação Carreira Ciências da Educação. Por um lado, um breve resumo das principais teorias feministas e sua relação com a educação, onde a ênfase é sobre ecológica e comunidade feminismos diz. Além disso, a experiência tem dois aspectos, um cognitiva que responde a aprender as teorias feministas, e o segundo uma pesquisa co-participativa em que o método biográfico foi usado para recuperar testemunhos, experiências e experiências de desenvolvimento Mulheres indígenas latino-americanas em suas lutas pelos territórios e pela natureza. Os principais resultados referem-se à sala de aula da universidade pode criar espaços de discussão e teorias feministas de diálogo e realização de pesquisas, o que mostra que a educação formal de uma educação da comunidade feminista pode ser um lugar de transformação e desconstrução os sistemas patriarcal e neocolonial. Uma das considerações finais que podem ser mencionados é que nas salas de aula constituem espaços em que a consciência pode levar ao empoderamento e de quebra círculos e conhecimentos opressão patriarcal.

Palavras-chave: Teorias Feministas e Educação, Feminismo Ecológico, Feminismo Comunitário, Método Biográfico, Pedagogia Comunitária Feminista, Mulheres Indígenas Latinoamericanas. 


\section{Introducción}

El presente escrito describe $\mathrm{y}$ sistematiza la experiencia de formación e investigación en teorías feministas con estudiantes de las asignaturas: Sociología General y Sociología de la Educación, (paralelo A) durante el calendario académico 2017 de la carrera Ciencias de la Educación, Universidad Mayor de San Andrés (UMSA) de la ciudad de La PazBolivia, la cual se debe a razones internas y externas. ${ }^{\mathrm{i}}$

Las razones internas hacen referencia al escenario de la carrera. Por un lado, la población estudiantil femenina es mayor a la de los varones, por ejemplo, en las materias mencionadas en el primer semestre se inscribieron 61 estudiantes, de los cuales el $14 \%$ varones y el $86 \%$ mujeres; en el segundo semestre se inscribieron 47 , de este total el $30 \%$ varones y el $70 \%$ mujeres. Por otro, la carencia de asignaturas y contenidos mínimos en el plan de estudios, actualmente se tiene un seminario sobre género y educación popular que está dirigido a estudiantes que realicen la preespecialidad de educación alternativa y popular, y no así para toda la población estudiantil. ${ }^{\text {ii }}$

En el caso de la investigación la mayoría de las tesis, proyectos de grado y trabajos dirigidos de licenciatura se focalizan más en el estudio de la pedagogía, didáctica y estrategias de aprendizaje, que, en problemáticas sociales relacionadas con la educación, por ejemplo, género, feminismos, cambio climático, medio ambiente, entre otros, aún falta profundizar y ampliar las miradas hacia la sociedad. También existe un fuerte arraigo tradicional del enfoque cuantitativo y de su pretendida neutralidad y objetividad del paradigma racionalista, aún falta por recorrer un largo camino para que se atrevan a dialogar con el enfoque cualitativo, los diferentes paradigmas $\mathrm{y}$ métodos de investigación.

En las razones externas se tienen dos aspectos, el primero, en Bolivia la violencia hacia las mujeres y feminicidios es una problemática vigente, pese a la existencia de la ley Integral para Garantizar a las Mujeres una Vida Libre de Violencia $\mathrm{N}^{\circ} 348$, por ejemplo, en la gestión 2016 se tuvieron 104 casos de feminicidio, en la gestión 2017109 y 37 en el primer trimestre del presente año. El segundo, es por el escenario de las movilizaciones de los pueblos indígenas de tierras bajas de Bolivia, quienes desde el 2011 iniciaron la protección del Territorio Indígena y Parque Isíboro-Sécure (TIPNIS) contra la construcción de una carretera, luego la defensa de la ley 180 
que protege al TIPNIS y en contra de la ley que anula su intangibilidad, -hoy vigente y sancionada-.

Estas son las razones que orientaron a reflexionar en el aula sobre el rol y participación de las mujeres indígenas bolivianas y latinoamericanas que se han manifestado a través de las protestas y marchas en contra de proyectos y políticas extractivistas que tienen como fin afectar la convivencia e interrelación con la naturaleza. "Nosotras como mujeres que pensamos en el futuro de nuestros hijos defenderemos nuestra CASA GRANDE porque es ahí donde nos reproducimos, ponemos en práctica nuestras culturas, ahí nos organizamos y vivimos en armonía con la naturaleza...". (Subcentral de Pueblos Indígenas: Mojeños-Yuracaré-Chimanes del TIPNIS, 2017, p. 2). Por ejemplo, en la VIII Marcha por la defensa del TIPNIS participaron cerca de 400 mujeres marchistas, mientras en la IX no se tienen datos cuantitativos que ofrezcan esta información.

Las mujeres suelen estar invisibilizadas en las movilizaciones porque generalmente son las figuras masculinas las que salen a la prensa o se piensa que el esfuerzo y coraje es propio de los varones. Para que la historia no quede incompleta, es importante personificar el papel de ellas en la figura de algunas mujeres que son una muestra de las muchas que fueron parte activa de la VIII Marcha por la defensa del TIPNIS (Huanca, 2012, p. 27).
Como parte de los contenidos de las asignaturas se desarrollaron varias unidades temáticas sobre las teorías feministas y educación, y para la investigación co-participativa el método biográfico permitió recuperar testimonios, experiencias y vivencias de mujeres indígenas latinoamericanas en sus luchas ambientales por los territorios y naturaleza. Esta forma de abordar los feminismos desde los contenidos curriculares e investigación; por un lado, fue para interiorizar y generar un proceso de concientización y sensibilización frente a los mecanismos de reproducción del sistema hetero-patriarcal en el ámbito educativo y en la sociedad boliviana; por otro, para recuperar y visibilizar las voces, saberes y epistemes de mujeres indígenas latinoamericanas.

El artículo se inicia con una descripción breve de las principales teorías feministas y su relación con la educación, enfatizando en los feminismos ecológico y comunitario. Posteriormente, se despliega la experiencia de formación e investigación para ello se describe el contexto, los participantes, el método, las fases, técnicas $y$ resultados que fueron los ensayos biográficos de mujeres indígenas latinoamericanas realizadas por las y los estudiantes. Luego, se aborda las 
consideraciones finales y las referencias bibliográficas.

\section{Teorías feministas y educación}

Según Madoo y Niebrugge-Branlley (1997)

el feminismo empieza en el momento en que comenzó la subordinación de las mujeres. Para González (1999) es un movimiento social e intelectual que se hace presente en la sociedad en muy distintas instancias sociales, políticas, científicas y educativas. Rodríguez (2002) señala que es un cuerpo de doctrina, una disciplina con textos fundacionales que ha impregnado la literatura, la historia, la filosofía, la ciencia, el arte. ${ }^{\text {iii }}$ Galindo (2010) desde otra perspectiva señala que el feminismo es una respuesta rebelde de insubordinación personal o colectiva de las mujeres frente y contra los mandatos patriarcales en cualquier lugar del mundo y de manera simultánea.

Con referencia a la educación al ser uno de los agentes de socialización, por un lado, reproduce y trasmite códigos culturales que refuerzan las estructuras de dominación y opresión patriarcal; por otro, puede transformar o deconstruir el sistema patriarcal. En este sentido, se tiene una diversidad de teorías feministas que toman sus posiciones del rol de la educación, por ejemplo, están el feminismo liberal, radical, socialista marxista, posestructuralista, postcolonial, negro $\mathrm{y}$ movimiento Queer (Ver Tabla 1).

Tabla 1. Principales teorías feministas y su relación con la educación.

\begin{tabular}{|c|c|c|}
\hline Teorías feministas & Miradas a la educación & Rol de la educación \\
\hline Liberal & $\begin{array}{l}\text { Desigualdad y discriminación de la } \\
\text { mujer consecuencia de procesos de } \\
\text { socialización, por ejemplo, la escuela. }\end{array}$ & $\begin{array}{l}\text { Igualdad, participación y movilidad } \\
\text { social es por el acceso a la educación. }\end{array}$ \\
\hline Radical & $\begin{array}{c}\text { Espacio de opresión que legitima y } \\
\text { reproduce las estructuras y relaciones } \\
\text { de dominación del poder hegemónico } \\
\text { masculino patriarcal, las "escuelas se } \\
\text { construyen sobre la subjetividad } \\
\text { masculina." }\end{array}$ & $\begin{array}{l}\text { La educación debe visibilizar las } \\
\text { experiencias, vivencias, valores y } \\
\text { modos de pensar y sentir de las } \\
\text { mujeres como medio para la } \\
\text { recuperación de su historia y su } \\
\text { cultura la cual está marginada y } \\
\text { silenciada por el saber patriarcal } \\
\text { (González, 2010). }\end{array}$ \\
\hline $\begin{array}{l}\text { Socialista } \\
\text { marxista }\end{array}$ & $\begin{array}{c}\text { El origen de desigualdad y opresión es } \\
\text { el producto de la división social y } \\
\text { sexual del trabajo. }\end{array}$ & $\begin{array}{l}\text { Permanente resistencia al control } \\
\text { social imperante. }\end{array}$ \\
\hline $\begin{array}{c}\text { Post- } \\
\text { estructuralista }\end{array}$ & $\begin{array}{c}\text { Incorporó en el análisis las relaciones } \\
\text { entre el poder, el cuerpo y la } \\
\text { sexualidad como mecanismos de } \\
\text { subordinación y dominación de las } \\
\text { mujeres. }\end{array}$ & $\begin{array}{l}\text { Resistencia al orden normativo a } \\
\text { partir de una praxis pedagógica } \\
\text { construida desde y con los y las } \\
\text { actoras/es que conforman el espacio } \\
\text { educativo (González, 2010). }\end{array}$ \\
\hline Post-colonial & $\begin{array}{l}\text { Procesos de dominación vinculada a la } \\
\text { colonización europea, al } \\
\text { neocolonialismo y al capitalismo. } \\
\text { La educación se constituye en un }\end{array}$ & $\begin{array}{c}\text { Deconstruir la educación mediante la } \\
\text { des-colonización del saber patriarcal } \\
\text { que se transmite y socializa en las } \\
\text { escuelas. }\end{array}$ \\
\hline
\end{tabular}




\begin{tabular}{|c|c|c|}
\hline & $\begin{array}{l}\text { aparato ideológico que reproduce las } \\
\text { condiciones coloniales y neocoloniales. }\end{array}$ & \\
\hline Negro & $\begin{array}{l}\text { Surge para reafirmar la cultura africana } \\
\text { se rechaza la pretensión de la } \\
\text { educación de desconocer la cultura y } \\
\text { las manifestaciones identitarias. }\end{array}$ & $\begin{array}{c}\text { La educación debe adecuarse a los } \\
\text { diversos contextos étnicos y } \\
\text { culturales. }\end{array}$ \\
\hline $\begin{array}{c}\text { Movimiento } \\
\text { Queer }\end{array}$ & $\begin{array}{l}\text { La educación no dispone de categorías } \\
\text { que comprendan la sexualidad en toda } \\
\text { su dimensión, su respuesta por lógica } \\
\text { es la normalización y el } \\
\text { disciplinamiento de los cuerpos. } \\
\text { El currículo esta conceptualizado para } \\
\text { excluir y castigar la diversidad sexual, } \\
\text { étnica y social. }\end{array}$ & $\begin{array}{l}\text { Coloca la discusión en torno a las } \\
\text { relaciones entre el sexo biológico, el } \\
\text { género y la orientación sexual, las } \\
\text { cuales deben ser incluidas y } \\
\text { valoradas en los procesos educativos. } \\
\text { Una educación no sexista. }\end{array}$ \\
\hline
\end{tabular}

Fuente: elaboración propia 2017, en base a Madoo \& Niebrugge-Branlley, 1997; González, 1999; Heras, 2009; González, 2010.

De las teorías mencionadas cada una tiene sus contribuciones y miradas de la situación de las mujeres, así como del rol que debe asumir la educación. Sin embargo, se debe mencionar que el feminismo liberal tiene como prioridad realizar cambios en la educación, por ejemplo, garantizar a las mujeres su acceso como derecho, por lo que, no busca trasformar y cuestionar las estructuras y relaciones patriarcales que se anclan en el sistema educativo como un aparato ideológico y simbólico de dominación masculina patriarcal. El feminismo socialista marxista si bien contribuye a comprender que la división sexual del trabajo fue por la aparición de la propiedad privada y con ella las clases sociales, su mirada del campo educativo se restringe a solo como un espacio de resistencia.

Se debe reconocer los aportes de las demás teorías para comprender la situación de la mujer: su diferencia, desigualdad y condición de grupo oprimido y su relación con la educación, este último es básicamente como un agente de socialización que reproduce las relaciones de dominación patriarcal. Por ejemplo, como campo de poder hegemónico masculino (F. Radical), un sistema educativo que niega las subjetividades, que excluye, violenta, reprime y anula (F. Posestructuralista), un aparato ideológico que reproduce las condiciones coloniales y neocoloniales (F. Postcolonial), desconoce las identidades y culturas (F. Negro) y un espacio de exclusión que castiga la diversidad sexual, étnica y social (M. Queer).

En el rol de la educación existen propuestas que establecen que debe constituirse en un espacio de resistencia (Socialista-Marxista y Posestructuralista) y de transformación (Radical, Postcolonial, Negro y Queer). Por ende, la educación y el sistema educativo son sitios donde se 
debe visibilizar la voz, experiencias, historias y subjetividad femenina (Radical), una praxis pedagógica construida desde y con los y las actoras/es que conforman el espacio educativo (Posestructuralista), una deconstrucción del saber patriarcal (Postcolonial), una interculturalidad y reconocimiento de la diversidad cultural (Negro), sexual y social (Queer). Pero, se necesita avanzar a comprender a la educación como un campo de poder simbólico y cultural, en el que se puede generar resistencia y enfrentar el poder, el transformar a nivel de las aulas desde la cotidianidad y práctica educativa. Como sugiere Botella (1991), una profunda reorganización de la estructura curricular, políticas educativas generosensitivas y un cuerpo docente formado para los desafíos que genera la educación en estos tiempos de crisis global.

\section{La emergencia de los feminismos ecológico y comunitario}

Svampa (2015) sugiere que dentro del ecofeminismo se tienen al diferencialista o identitario, que naturaliza la relación entre mujer y naturaleza; el constructivista, que concibe esa relación como una construcción histórico social, ligada a la división sexual del trabajo. En este sentido, se tiene diferentes matices y cuerpos conceptuales de estos feminismos, los cuales fueron identificados por la investigación co-participativa realizada con las y los estudiantes de las asignaturas, el cual sirvió para plasmar los ensayos biográficos de mujeres indígenas latinoamericanas.

En el caso del feminismo ecológico como ejes comunes plantea la defensa del medio ambiente, del territorio y de la naturaleza, y liga el ecologismo con el feminismo. Se tienen diferencias al interior de esta corriente, una de ellas es la crítica al mal desarrollo, al modelo económico y cultural occidental que se caracteriza por los postulados patriarcales modernos de homogeneidad, dominación y centralización realizada por Vandana Shiva en Abrazar la vida (1995). Según Shiva, el enemigo no es el varón, sino el capitalismo patriarcal del colonizador, por ello, pondrá en evidencia la existencia de un "patriarcado capitalista."

La subordinación de las mujeres a los hombres y la explotación de la naturaleza son dos caras de una misma moneda y responden a unas lógicas comunes: la ilusión de poder vivir al margen de la naturaleza, el ejercicio del poder patriarcal y del sometimiento de la vida a la exigencia de la acumulación. (Shiva \& Mies, 1997, p. 8).

De acuerdo a Svampa (2015) está ligado a la corriente de la ecología popular, y que algunos denominan "ecofeminismo 
de la supervivencia”, ya que estaría vinculado a la experiencia diversa de las mujeres en la defensa de la salud, la supervivencia, y el territorio.

Por otra parte, Alicia Puleo propone una visión histórica que se inicia con el ecofeminismo clásico. Para Puleo (2013) el aporte de Mary Daly Gyn/Ecology (1978) será uno de los primeros en plantear un feminismo ecológico. De acuerdo a Svampa (2015) las contribuciones de Puleo provienen de las crisis ecológica entendida como una crisis social de carácter antropológico: la necesidad del dominio como fórmula para la afirmación de lo humano se reflejaría en el plano de las relaciones interpersonales y en el vínculo de lo humano con lo natural. Puleo (2011) menciona que el feminismo y el ecologismo permiten realizar una mirada distinta sobre la realidad cotidiana, revalorizando aspectos, prácticas y sujetos que habían sido designados como diferentes e inferiores. Puleo interpela el "ecofeminismo de la supervivencia," así como la especificidad femenina de las tareas del cuidado. "Hay que universalizarlas, enseñar actitudes, virtudes y prácticas del cuidado a los varones ya que todos los seres humanos podemos desarrollarlas. Y es necesario extender el cuidado humano desgenerizado a la naturaleza no humana". (Puleo, 2013, p. 40).

En lo referido al feminismo comunitario, tiene su propia raíz histórica, vivencia, contexto y diferencias, aunque Puleo (2013) lo incluye como parte del ecofeminismo denominándolo espiritual que surge en Latinoamérica y se caracteriza por su interés en las mujeres pobres, su defensa de los indígenas, víctimas de la destrucción de la naturaleza, y su crítica a la discriminación de la mujer en las estructuras de autoridad religiosa.

Por un lado, se tiene la crítica al feminismo occidental de igualdad y de la diferencia realizada por Julieta Paredes quien propone que el comunitario surge de la propia experiencia e historia indígena andina. "Nosotras partimos de la comunidad como principio incluyente que cuida la vida. Para construir el feminismo comunitario es necesario desmitificar el chacha-warmi (hombre-mujer) que nos impide analizar la realidad de la vida de las mujeres en nuestro país [Bolivia]". (Paredes, 2010, p. 27). Su propuesta se centra en el par complementario horizontal que es su reconceptualización: “... despojarlo de su machismo, de su racismo y su clasismo, replantearlo en mujerhombre, warmi-chacha que recupera el par complementario horizontal, sin jerarquías, armónico y recíproco, par de presencia, 
existencia, representación y decisión”. (Paredes, 2010, p. 30); el reconocimiento de la existencia real de las mujeres como parte de la comunidad: "Reconocer a las mujeres es construir otro discurso ahora incluyente y real. Al decir que la comunidad está compuesta por las mujeres y los hombres visibilizando a las mujeres invisibilizadas por la hegemonía de los hombres". (Paredes, 2010, p. 34). Por lo tanto, el patriarcado es el sistema de las opresiones, discriminaciones y violencias que vive la humanidad (mujeres, hombres y personas intersexuales) y la naturaleza. En este sentido, se apunta hacia una descolonización y des-patriarcalización.

Por otro, Lorena Cabnal (2010) propone la recreación y creación de un pensamiento político ideológico feminista que reinterprete las realidades de la vida histórica y cotidiana de las mujeres indígenas dentro del mundo indígena. Cabnal, tomando las ideas fuerza de Paredes y Shiva, plantea que existe un patriarcado originario ancestral, una penetración colonial y el entronque de patriarcados, una construcción del mundo masculino, una victimización histórica situada en un racismo. Su propuesta es la recuperación y defensa de "nuestro territorio cuerpo-tierra" y una cosmovisión liberadora “...de la opresión histórica contra los cuerpos sexuados de mujeres y contra la opresión histórica capitalista contra la naturaleza, pero a su vez evocan e invocan las resistencias y transgresiones ancestrales de las mujeres". (Cabnal, 2010, p. 24).

Un balance que se puede realizar es que ambas corrientes feministas tienen diferentes posiciones $\mathrm{y}$ aportes. Por un lado, el ecológico plantea la defensa del medio ambiente, del territorio y de la naturaleza, la presencia de un "patriarcado capitalista" (Shiva) y rescate de la cultura del cuidado humano hacia la naturaleza no humana (Puleo), estas propuestas amplían la posibilidad de "... pensar una sociedad ecológica y socialmente sostenible, a través de valores como la reciprocidad, la cooperación y la complementariedad". (Pascual, 2010 citado por Svampa, 2015, p. 131). En suma, los aportes mencionados buscan visibilizar el protagonismo de las mujeres como sujetos históricos y políticos que defienden sus territorios ante la expansión del capitalismo y frente a la crisis ambiental.

Por otro, el comunitario, coincide en plantear un patriarcado originario ancestral y una penetración colonial, postcolonial y capitalista, pero se tienen diferencias $\mathrm{y}$ miradas distintas, por ejemplo, Paredes parte de una crítica al feminismo occidental como una construcción foránea a la realidad sociocultural de los pueblos 
indígenas latinoamericanos. Asimismo, sus propuestas de deconstrucción del sistema patriarcal y de la educación, tienen rasgos diferentes, en el caso de Cabnal plantea la recuperación y defensa del cuerpo-tierra y contra la opresión histórica capitalista debe ser desde las mujeres; mientras que Paredes propone partir de la comunidad y del par complementario horizontal (mujerhombre) para el reconocimiento y visibilización real de las mujeres como parte de la comunidad. Esta última propuesta abre las puertas para que la comunidad, las mujeres y los hombres en complementariedad y reciprocidad puedan ser parte activa de la deconstrucción del sistema patriarcal, el despojo del machismo, racismo, saber patriarcal y exclusión.

\section{Pedagogía comunitaria feminista: el aula como espacio de resistencia $y$ transformación}

La educación y el sistema educativo pueden ser a su vez un espacio de transformación, pero también de adoctrinamiento, reproducción y legitimación de mensajes de opresión, de sometimiento y de desigualdad. Mientras la pedagogía es un discurso que contextualiza y orienta la educación, “sustenta y promueve la ética y un objetivo político, y que como tal, es una herramienta indispensable para el trabajo y avance del feminismo". (Maceira, 2008, p. 28). Para Montenegro (2016) el discurso pedagógico es también un posicionamiento que, en el caso de las pedagogías feministas, se plantea desde la distancia con el patriarcado y en actitud propositiva, colaborativa e integradora, logrando formas de ver las pedagogías que no se limitan al quehacer de las escuelas formales.

Las pedagogías feministas pueden ser pensadas en sentidos más amplios que permean la escuela, el colegio y las aulas universitarias. Como sugiere González (2010) en el aula se puede lograr cambios significativos es cuestión de formación y compromiso social para poder ir impactando el orden de género, el régimen de género y la ideología de género en las prácticas cotidianas educativas.

\section{Según Belausteguigoitia y Mingo} (1999) las pedagogías feministas nacen del descontento de ver cómo el patriarcado, entendiendo el término como una situación o panorama de desigualdad entre hombres y mujeres en diversos ámbitos de la vida social, donde los primeros tienen privilegios y situaciones de control sobre las segundas. Panorama que se apoderó de los procesos de escolarización, manteniendo y reproduciendo discursos masculinistas en las aulas. Segato (2016) señala que las relaciones entre los géneros 
se organizan en términos de jerarquización donde lo masculino está en una posición de poder sobre lo femenino. En este sentido, la pedagogía asociada al feminismo busca deconstruir la educación tradicional y reproductora de las desigualdades y opresión.

Para Gargallo (2008) la mirada feminista permite deconstruir los mandatos de género hetero-normativos. "Hablar de una pedagogía feminista es hablar de interseccionalidad, de multidimensionalidad, de la inclusión de las diferencias y de transformación social. Es diálogo, compresión, colectividad, educación popular, empoderamiento $\mathrm{y}$ ruptura de los círculos de opresión". (Martínez \& Ramírez, 2017, p. 85). De acuerdo a Sánchez, Penna y de la Rosa (2016) educar desde el feminismo debe ser un acto amoroso de respeto a cada persona en sus diferencias. A decir de Gonzáles (2010) se debe transformar la educación como espacios de empoderamiento y por ende de respeto y reconocimiento a las diferencias.

En este sentido, las niñas y niños necesitan aprender a pensar, a hablar, a sentir y a actuar desde el respeto a las diferencias. Todo este proceso de enseñanza y aprendizaje (construcciónde-construcción) tiene en la formación del profesorado un aliado poderoso (Martínez \& Ramírez, 2017, p. 84).
Es posible desde una pedagogía comunitaria y feminista generar la descolonización y des-patriarcalización de la educación tradicional que reprime la diversidad y diferencia. "Donde la masculinidad y la feminidad son definidas bajo unos mandatos rígidos $\mathrm{y}$ donde esa feminidad se pone al servicio de los privilegios de dicha masculinidad". (Martínez \& Ramírez, 2017, p. 82).

En Bolivia, la des-patriarcalización consiste en la elaboración de políticas públicas desde la identidad plurinacional, para la visibilización, denuncia $\mathrm{y}$ erradicación del patriarcado, a través de la transformación de las estructuras, relaciones, tradiciones, costumbres $\mathrm{y}$ comportamientos desiguales de poder, dominio, exclusión, opresión y explotación de las mujeres por los hombres (Asamblea Legislativa Plurinacional de Bolivia, ley 348, 2013, Art. 4, Numeral 12). Sin embargo, las políticas públicas no garantizan una des-patriarcalización plena y real, por ejemplo, el alto índice de violencia hacia las mujeres en Bolivia, pese a la existencia de una ley y políticas. Se necesita el protagonismo y empoderamiento de las mujeres, hombres y comunidad para desmontar el sistema de poder patriarcal. Para Galindo (2015) es el desmontaje de la estructura de poder, una metodología de práctica política que 
supone la capacidad de decodificar, desmontar, desmantelar los mandatos $\mathrm{y}$ estructuras patriarcales y el reconocimiento de un nuevo punto de partida.

El cual puede ser desde la misma cotidianidad, la educación formal (escuelas, colegios y aulas universitarias) y popular y alternativa, son espacios destinados a abrir diásporas de despatriarcalización y des-colonización, esta última implica recuperar y reescribir la historia oficial que niega el protagonismo y el saber de las mujeres: "la historia se centra en el hombre que representa el papel principal y la mujer es solamente una guinda del pastel" (Ortiz, 1992); el desmontar la sociedad androcentrista y su representación sociocultural de la mujer "asociada con la naturaleza, fecundidad, su rol de esposa, proveedora de cuidado y protección". (Iño, 2017, p. 8).

Galindo (2015) y Paredes (2010) reconocen que la des-colonización y la des-patriarcalización son matrices importantes de la lucha feminista postcolonial y comunitaria. "El patriarcado contiene a la colonización y no al revés...Afirmamos que la colonización es un instrumento del patriarcado y, por lo tanto, la des-patriarcalización comprende e incluye a la descolonización, convirtiéndose en una de sus tareas fundamentales". (Feministas Comunitarias de Abya Yala, Bolivia, 2016, p. 41).

Para Paredes (2010) y Zabala (2012) resignificar la educación bajo las claves de la des-patriarcalización y des-colonización implica: a) un feminismo comunitario como construcción de derechos individuales y colectivos; b) dar voz y poder a las comunidades indígenas, campesinas, excluidas; c) incluir las diferencias y las diversidades a partir de creativas formas de comunicación (educación popular, técnicas como el teatro-foro, asambleas, música, simbología, libros, cuerpos; d) creación de redes de sororidad (relación de hermandad y solidaridad) entre mujeres que favorezcan la toma de conciencia individual $y$ colectiva de las diversas opresiones y violencias; e) procesos de educación comunitaria y permanente para el empoderamiento.

La experiencia que se desarrolló considero los aportes de las teorías feministas (radical, post-estructural, postcolonial, Queer y negro) y de los feminismos ecológico y comunitario. Por lo que, buscó resignificar la educación y praxis pedagógica en aulas universitarias como espacios en los que se pueden dialogar, discutir y debatir colectivamente; sensibilizar, concientizar y visibilizar las desigualdades de género, opresión y 
dominación de las mujeres. También para incidir en los contenidos, lenguajes, metodologías, evaluaciones, saberes y prácticas dominantes y tradicionales que se presentan en la formación de profesionales en Ciencias de la Educación de la UMSA.

Para ello se realizó una investigación co-participativa como una herramienta que permite desarrollar procesos de formación en las aulas desde una pedagogía comunitaria feminista. El método biográfico posibilitó visibilizar y reconocer la diversidad de saberes, experiencias, voces, formas de sentir e historias de vida de mujeres indígenas latinoamericanas que desplegaron sus luchas y defensas por el territorio y la naturaleza, esta última fue sistematizada por medio de ensayos biográficos.

\section{Investigando sobre teorías feministas desde el aula con estudiantes universitarios}

La experiencia se llevó a cabo en la carrera Ciencias de la Educación de la UMSA, específicamente con estudiantes de la asignatura Sociología General y Sociología de la Educación (paralelo A), el cual responde a la necesidad de introducir aprendizajes sobre las teorías feministas y procesos de investigación en el aula que tomen como campo de estudio a las mujeres. Ambos aspectos son importantes en la formación universitaria de los profesionales en Ciencias de la Educación.

Esta forma de generar aprendizajes responde al enfoque de la pedagogía crítica y transformadora, la cual establece la importancia de interrelacionar los aprendizajes al contexto y los problemas que se presentan. Asimismo, la educación puede generar su transformación y liberación, como también el posicionamiento ético y epistémico.

El avance de contenidos y la investigación co-participativa fue realizada como parte de los aprendizajes y enseñanzas de las asignaturas, esto evidencia que, ante la ausencia de materias dedicadas a abordar la pedagogía feminista, las asignaturas como Sociología, Antropología, Historia y Filosofía pueden generar diásporas de una pedagogía comunitaria feminista, como lo sucedido con esta experiencia: una forma de feminizar las aulas, a las y los estudiantes y al docente.

En el desarrollo del aprendizaje durante los dos semestres se dedicaron varias unidades temáticas que abordaron las relaciones de género y las teorías feministas, las cuales se interrelacionaron con el campo educativo. Las sesiones fueron en base a grupos de discusiones y debates en torno a autores y lecturas seleccionadas, el contexto boliviano y 
latinoamericano y la experiencia personal de las y los estudiantes. En las lecturas se realizaron sesiones de discusión y plenarias en las que se presentaron los enfoques de análisis que se interrelacionaron con el contexto boliviano: la situación de las mujeres y las movilizaciones por la defensa del TIPNIS.

Los propósitos fueron primero, comprender y analizar las distintas teorías feministas; profundizar en los feminismos ecológico y comunitario con el fin de articular con sus trabajos de investigación. Segundo, interrelacionar los contenidos conceptuales con la investigación para generar un aprendizaje significativo, vivencial y participativo. Tercero, aprender y situar los liderazgos de mujeres indígenas latinoamericanas en su defensa y lucha por sus territorios y naturaleza. Cuarto, visibilizar sus voces y discursos con la identificación de experiencias personales. Quinto, recopilar y sistematizar en ensayos biográficos las trayectorias de vida, vivencias y experiencias como una forma de visibilizar sus voces, discursos y saberes feministas. Todos los propósitos mencionados se orientaron a generar una apropiación y toma de conciencia y posición ética de las y los estudiantes y del docente.

\section{Método}

La investigación se desarrolló desde el enfoque cualitativo con el estudio exploratorio que abordo la temática de "Mujeres indígenas en Latinoamérica, su liderazgo en las luchas ambientales por los territorios y la naturaleza." La historia debe personificar el papel de ellas en algunas mujeres que son muestra de las muchas que luchan y defienden sus territorios y naturaleza. Se recurrió a la investigación biográfica porque permite estudiar $\mathrm{y}$ aprender de la historia y experiencia de vida de alguna persona que se sitúa en un tiempo histórico o coyuntura. Su estrategia metodológica se dirige a valorar el testimonio oral y voz, la memoria individual, también se puede recurrir al testimonio escrito.

La investigación co-participativa llevada a cabo tuvo distintas fases. 1) La primera, organización del equipo de trabajo, se inició con la definición del tema a investigar, método y técnicas, y la conformación de grupos de trabajo, para el primer semestre se tuvo catorce grupos: de los cuales cinco estaban integrados en su totalidad por mujeres, dos de solo varones y siete grupos mixtos (varones y mujeres); en el segundo semestre once grupos: cinco mixtos, cinco solo de mujeres y uno con integrantes varones. 
2) En la segunda fase, se llevó a cabo un estudio bibliográfico y documental con el empleo de la técnica del fichaje, se tuvieron dos momentos. i) En el primer semestre los diferentes grupos de trabajo recopilaron y sistematizaron información bibliográfica referente a los ejes temáticos: género, trabajo doméstico, cuidado, roles, derechos y educación, representación social de las mujeres y las narrativas de las diversas teorías feministas. La revisión y recopilación fue realizada en distintas bibliotecas de la cuidad de La Paz-Bolivia y en virtuales de México y Argentina. Esta información sirvió para delimitar el tema de investigación y conocer el estado de la producción bibliográfica. ii) En el segundo semestre se procedió; por un lado, a complementar la información referida a los feminismos comunitario y ecológico; por otro, recopilar fuentes de información sobre las líderes indígenas identificadas, se revisaron en la web, periódicos digitales, revistas académicas, blogs, testimonios, reportaje y entrevistas de canales de televisión. La sistematización de esta información se la realizó con la selección y clasificación de las fuentes en periódicos, documentales, reportajes y entrevistas en formato de video; en el caso de las notas de prensa fueron seleccionados de acuerdo a los datos que ofrecían, mientras los reportajes y entrevistas fueron transcritas.
3) La tercera fase, para el análisis de información, en las fuentes impresas se empleó la técnica del subrayado y las lecturas exploratoria y selectiva, en esta parte las fichas bibliográficas, de resumen $\mathrm{y}$ textuales fueron muy útiles para organizar las fuentes de información. Para las entrevistas se utilizó el análisis de contenido para la extracción de partes concretas, de acuerdo a cuatro niveles de lectura: i) intuitiva, para identificar los "titulares de prensa" en los discursos; ii) temática, secciona el discurso en temas y subtemas que se presentan; iii) contextual, para analizar los aspectos que rodean la acción: el contexto y el lugar; iv) y la relacional, contribuye a construir las partes del discurso e identificar las redes de cada entrevista.

4) La cuarta fase, fue la redacción del ensayo biográfico realizada sobre la base de seis aspectos: i) conocer las fechas de nacimiento y muerte, ii) obtener información de la familia e infancia, iii) analizar la formación y estudio, iv) señalar el contexto, v) conocer su actividad, v) influencia social o participación en su contexto, y vi) valorar su papel o contribución. Se llevaron a cabo sesiones colectivas de seguimiento, que fueron mediante exposiciones y presentación de avances, revisión y acompañamiento en la 
redacción del ensayo biográfico realizado en talleres participativos.

5) En la quinta fase se efectuó la socialización de los resultados de la investigación, que fue mediante un conversatorio titulado: "Diásporas del feminismo comunitario y ecológico: Mujeres indígenas en Latinoamérica, su liderazgo en las luchas ambientales por los territorios y la naturaleza" (ver anexo), en la que las y los estudiantes presentaron sus ensayos biográficos en calidad de expositores. Así también varios de ellos y ellas participaron en el programa "Aula Trece" del canal universitario de la UMSA para compartir sus aprendizajes, experiencias y vivencias respecto de sus trabajos de investigación.

\section{Resultados: Una pedagogía comunitaria feminista para aprender a des- patriarcalizar las aulas universitarias}

Los resultados que se pueden mencionar son los aprendizajes logrados como parte de la experiencia desarrollada, permiten establecer la posibilidad de construir una pedagogía comunitaria feminista para des-patriarcalizar las aulas universitarias. El primero, hace referencia a las miradas y comprensiones que se tuvieron en la fase de revisión bibliográfica, en donde se pudo apreciar bastante interés de parte de las y los estudiantes por conocer, aprender e investigar sobre las teorías feministas, relaciones de género, trabajo doméstico, representación social, entre otros. En las sesiones de debate y discusiones en las plenarias, los enfoques de análisis se interrelacionaron con sus experiencias personales y la situación de las mujeres en el contexto boliviano.

Con referencia a la discusión de los feminismos se logró entender sus complejidades y su interrelación con el campo educativo, y por qué como futuros profesionales en Ciencias de la Educación deben conocer $y$ formarse para poder comprender la situación de la mujer, el reconocer la presencia de un sistema patriarcal y una educación, un acto pedagógico (currículo y libros) y agentes (educadores) que pueden ser reproductores o transformadores de esa sociedad patriarcal. Es decir, asumir una posición ética de compromiso social frente a las problemáticas que se presentan en la comunidad.

También, llegaron a comprender el rol que tiene la educación, el sistema educativo, el currículo y los maestros/as, los cuales pueden ser espacios de invisibilización y reproducción del sistema patriarcal y subjetividad masculina $\mathrm{o}$ entornos de visibilización de las voces, historias, experiencias y subjetividad 
femenina. Por ejemplo, las y los estudiantes retomando las teorías feministas posestructuralista, postcolonial, negro y Queer entendieron que es posible desde las aulas y la cotidianidad realizar praxis pedagógicas de resistencia y transformación que deconstruyan el saber patriarcal.

En el caso de los feminismos ecológico y comunitario diferenciaron sus orígenes y corrientes, por ejemplo, los grupos hicieron una toma de posición al establecer que existen coincidencias, pero, a la vez diferencias entre ambas y al interior de cada corriente. Esto se evidencio en sus ensayos y exposiciones, los grupos se sustentaron en Shiva y Cabnal para fundamentar la visibilización real de los liderazgos de mujeres indígenas en la defensa de sus territorios y de la naturaleza; mientras que Paredes sirvió de referencia para entender que la liberación y emancipación de la opresión patriarcal, primero, es desde la mujeres con la coparticipación de hombres, y segundo desde la comunidad, estas conclusiones fueron discutidas largamente entre las y los estudiantes durante las plenarias y en el conversatorio. Asimismo, dio la posibilidad de visualizar que los hombres pueden ser parte de la liberación de opresión y dominación que ejerce el sistema patriarcal. Por ejemplo, en los grupos integrados por solo varones, estos en las primeras plenarias realizaban comentarios y chistes machistas, pero conforme se realizaban las actividades de formación e investigación, se dieron cuenta que no era la forma correcta y ética de actuar y que debían generar aperturas de respeto y tolerancia hacia las mujeres.

El segundo, es la contribución a su formación como profesionales en Ciencias de la Educación, el incorporar esta temática como parte de sus aprendizajes generó y despertó el interés de parte de las y los estudiantes de realizar análisis de la realidad boliviana y latinoamericana respecto de la situación de la mujer, representación, roles, luchas e historia. A la vez, se puso en evidencia que la educación necesita interactuar con otras ciencias o disciplinas para poder dar respuestas a las problemáticas sociales, por ejemplo, el tema de violencia hacia las mujeres.

Específicamente en su formación, en todo el ciclo de las materias se dieron momentos de aprendizaje significativos, porque ellos, fueron los actores $\mathrm{y}$ protagonistas activos, por ejemplo, conocieron y comprendieron la situación de las mujeres en cuanto su diferencia, desigualdad, opresión y dominación por el sistema patriarcal; escribieron la vida de mujeres indígenas, a través de sus 
investigaciones y ensayos biográficos, evidenciaron el poder simbólico y cultural que tiene el conocimiento para visibilizar la vida, historia, experiencias, voces $\mathrm{y}$ saberes de las mujeres, así como la necesidad de tener una posición ética.

El tercero, es el referido al empleo del método biográfico en la educación, en este caso en la investigación que realizaron y sus resultados la redacción y presentación de los ensayos biográficos de mujeres indígenas latinoamericanas. El recurrir a la historia de vida permitió que las experiencias y saberes propios de las y los estudiantes interactúen con las vivencias y voces de las mujeres indígenas, es decir, permitió acercarse a una realidad específica y a una experiencia individual concreta.

Un balance que se puede realizar de los resultados de la investigación es que fueron plasmadas en ensayos biográficos que reflejan que cada mujer indígena representa un contexto diferente, pero que tiene un escenario similar, su situación y condición de ser mujer indígena en una sociedad patriarcal, así como su protagonismo y liderazgo en la defensa de sus territorios y de la naturaleza. Por un lado, se tiene a líderes que asumen una posición de generadoras de saberes y de epistemologías propias y feministas que emergen de sus experiencias, vivencias y voces, unas desde la des-patriarcalizacion, el par complementario horizontal (mujereshombres) de Julieta Paredes, Blanca Chancoso y Aída Quilcué; otras desde la defensa de la tierra y la opresión histórica capitalista de Lorena Cabnal y el cuidado del medio ambiente en Betty Cariño (+) y la defensa de derechos de los indígenas en Berta Cáceres (+).

Por otro, líderes que emergen desde un protagonismo ligado a la defensa de su territorio frente a la destrucción de la naturaleza, es decir, surgen de las circunstancias y coyunturas que se presentan en sus comunidades como el caso de Francisca "Chica" Ramírez y su defensa del territorio ante la construcción del "Canal Interoceánico" en Nicaragua; Flor María Paraná frente al derrame de petróleo del río Marañón en el Perú; Patricia Gualinga en la Amazonia ecuatoriana su defensa ante la explotación petrolera; Máxima Acuña y la lucha por sus tierras en el altiplano peruano $\mathrm{y}$ Millaray Huichalaf defensora de los espacios sagrados Mapuches ante la construcción de la central hidroeléctrica en Chile.

El cuarto, es la importancia que tiene el aprender de la vida e historia de mujeres indígenas para sentir y posicionar una pedagogía comunitaria feminista en las aulas universitarias. El escribir su historia 
de vida en ensayos biográficos permitió una apropiación e internalización, el sentir lo emocional desde lo racional. La investigación en noticias de prensa, escrita, artículos en revistas, reportajes, entrevistas, testimonios y voces de los protagonistas, posibilitó una concientización y valoración de los estudiantes de la realidad, problemas, contextos, dificultades y contribuciones que realizaron las mujeres indígenas latinoamericanas investigadas.

Esto fue porque en varios de los grupos de trabajo no solamente llegaron a conocer y describir, sino palparon $\mathrm{y}$ sintieron sus vivencias y experiencias de vida, el cual fue reflejado en las exposiciones que realizaron durante los días del conversatorio, en donde se vio la apropiación de las historias de vida, su indignación por los asesinatos de Caceres y Cariño, por las injusticias, inequidades, desigualdades, opresión y dominación que viven las mujeres indígenas. Por ejemplo, cada grupo al final de su ensayo biográfico realizó una valoración de la contribución de las líderes mujeres indígenas a su contexto, a la acción política e episteme feminista (Ver Tabla 2).

Tabla 2. Percepciones y conclusiones de los grupos de trabajo de investigación.

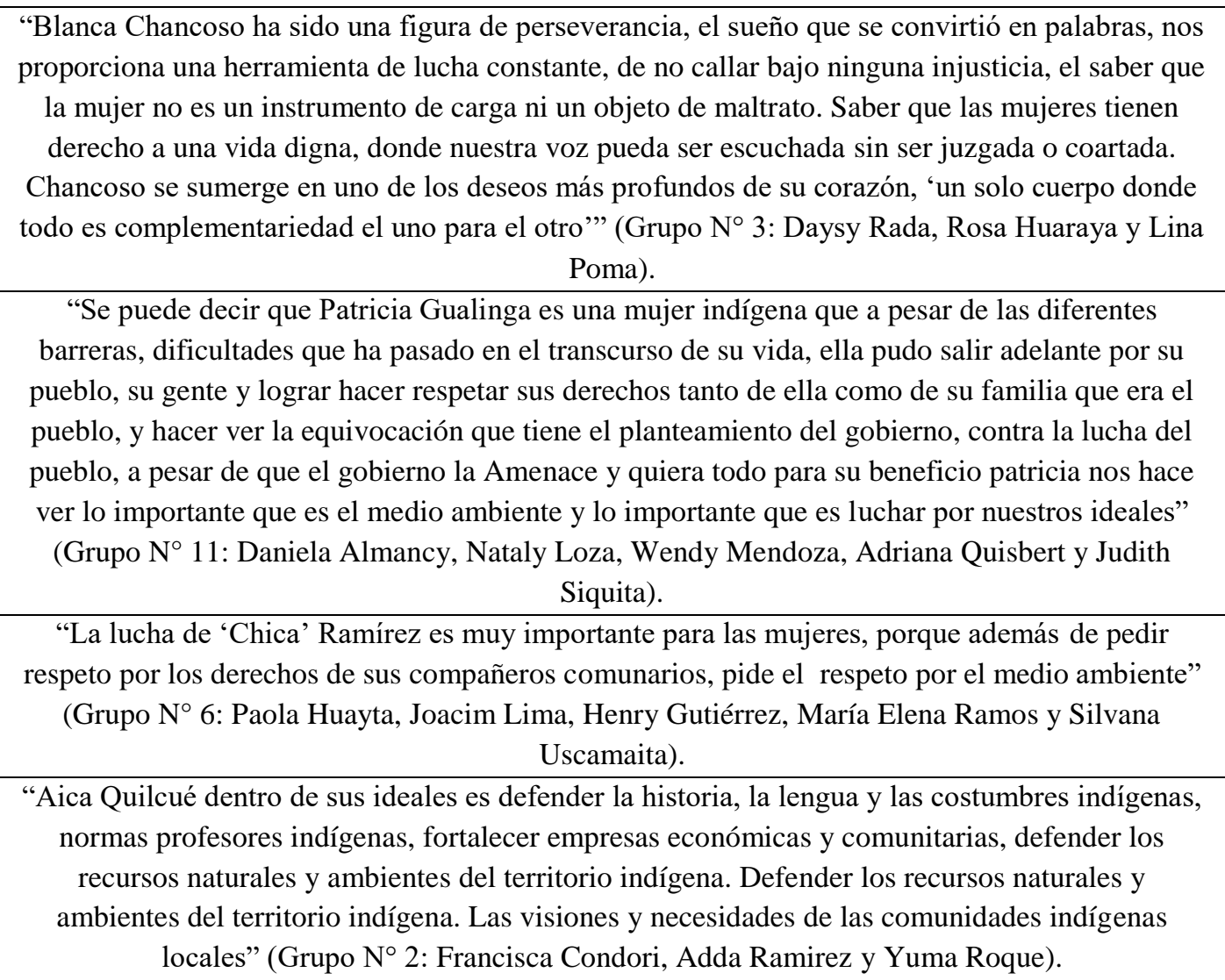




\begin{tabular}{|c|}
\hline "Millaray Huichalaf fue vocera para dar a conocer su situación de lo que estaba pasando su \\
territorio. Donde fue hostigada, criminalizada y fue presa política por diferentes motivos, pero \\
más que todo fue humillada como mujer y como madre, Frente a esta lucha se vulneraron sus \\
derechos de igualdad porque su religiosidad fue afectada por la inundación de su sitio ceremonial \\
y querían quitarles el derecho de poder vivir en un medio ambiente libre de contaminación" \\
(Grupo N 4: Elizabeth Castillo, Anahí Mendoza y Grecia Montecinos). \\
"Máxima Acuña es un gran ejemplo de lucha para todas las mujeres que necesitan ser \\
escuchadas" (Grupo No 1: Marina Apaza, Yoli Flores, Pamela Alberto y Jheanette Copa). \\
\hline "Flor María Paraná es una mujer indígena líder que no se ha rendido y su lucha continua" (Grupo \\
$\mathrm{N}^{\circ}$ 11: Daniela Almancy, Nataly Loza, Wendy Mendoza, Adriana Quisbert y Judith Siquita). \\
\hline
\end{tabular}

Fuente: elaboración propia 2017, en base a ensayos biográficos de mujeres indígenas latinoamericanas.

\section{Consideraciones finales}

La educación y sistemas educativos en este siglo XXI necesitan una reorientación hacia su comprensión como espacios de transformación y deconstrucción, en donde el discurso de la pedagogía crítica y transformadora dialogan con las pedagogías comunitarias feministas para generar una deconstrucción de la educación tradicional y reproductora de las desigualdades y opresión, hacia el respeto y reconocimiento de las diferencias y diversidades.

La experiencia descrita y sistematizada buscó abrir espacios de diálogos colectivos entre estudiantes y el docente en el tema de las desigualdades de género, situación de las mujeres, opresión, dominación y exclusión, teorías feministas y educación desde el aprendizaje de contenidos y la realización de investigaciones. Estas revisiones históricas permiten apreciar que en Latinoamérica se viene desplegando feminismos desde las calles y territorios que tienen como eje de lucha la defensa del medio ambiente, agua y territorio, porque, estos recursos son vitales para la pervivencia de la vida. Es decir, aparte de los aportes teóricos en Latinoamérica emergen epistemes y saberes locales feministas lideradas por mujeres indígenas.

En este sentido, la formación universitaria en carreras como educación requieren generar una toma de conciencia, de posición ética y epistémica con respecto a la construcción social del patriarcado en el campo educativo. Es decir, se necesita humanizar, concientizar y sensibilizar a estudiantes y docentes sobre la necesidad de deconstruir estos espacios de socialización, es atreverse a desplegar pedagogías comunitarias feministas en las aulas universitarias con el propósito de lograr cambios significativos y constituir esos escenarios educativos como espacios de empoderamiento, respeto $\mathrm{y}$ reconocimiento de las diferencias y diversidades. 
Iño, W. G. D. (2018). Teorías feministas en el aula: una experiencia de formación e investigación con estudiantes de Ciencias de la Educación...

Anexo: Programa de conversatorio.

UNIVERSIDAD MAYOR DE SAN ANDRÉS CARRERA CIENCIAS DE LA EDUCACIÓN

TALLER DE HISTORIA ORAL ANDINA

Organizan: Estudiantes de la materia de Sociología de la Educación, 1er. Año

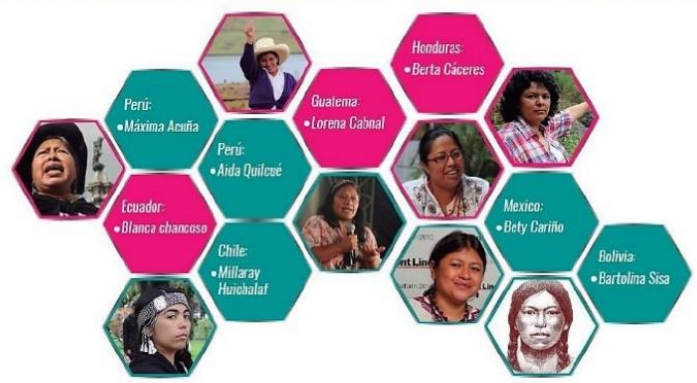

Conversatorio:

Diásporas del feminismo comunitario y ecológico: "Mujeres indígenas en Latinoamérica, su liderazgo en las luchas ambientales por los territorios y la naturaleza"

MARTES 05 DE DICIEMBRE, TALLER DE HISTORIA ORAL ANDINA

Diásporas del feminismo comunitario y ecológico. Lic. Weimar Iño

Feminismo comunitario, todas somos parte de un todo. Universitarixs: Pamela Ancon, Edwin Vargas,

Nataly Choque Fuentes y Andrea Ayoroa Monrroy

Matices del ecofeminismo. Universitarixs: Ana Cristina Huayhua, Eduardo Cruz, Saúl Crespo y

Ximena Santa Mamani Patty

10:00

Lorena Cabnal: Mi cuerpo, mi territorio libre de patriarcado. Universitarixs: Jhonatan Huanca,

Adhemar Azcui Chávez, Boris Arcil Condori, Wendy Soria Soto y Mayra Fernández

Ronda de preguntas y comentarios

10:30

Refrigerio

11:00

Moderador y comentarista. Lic. Rodolfo Quisbert

Blanca Chancoso y un solo cuerpo. Universitarixs: Daysy Rada Zapata, Rosa Isela Huaraya Villca y

Lina Poma Hanara

$11: 30$

Flor María Paraná: “No quedo nada, nada." Universitarixs: Daniela Almancy Lopez, Nataly Loza D., Wendy Mendoza Nina, Adriana Quisbert Yanarico y Judith Siquita Saravia

Ronda de Preguntas y comentarios

$12: 10$

$12: 40$

JUEVES 07 DE DICIEMBRE, TALLER DE HISTORIA ORAL ANDINA

Moderador y comentarista. Univ. Yessenia Valenzuela

Mi pueblo, mi vida, la lucha sigue: Aida Marina Quilcue Vivas. Universitarixs: Francisca Condori Cutipa,

Adda Luz Ramirez Zanabria y Yuma Roque Espinoza

08: 30

Patricia Gualinga: "Yo no decidí ser líder, la circunstancia me obligo a serlo". Universitarixs:

Daniela Almancy Lopez, Nataly Loza, Wendy Mendoza, Adriana Quisbert y Judith Siquita 09:00 Mi nombre es Millaray Huichaluf, "yo soy machi, el doble Carimallin." Universitarixs: Elizabeth Castillo,

Anahí Mendoza Fernández y Grecia Montecinos Rojas 09:30 $\begin{array}{ll}\text { Ronda de preguntas y comentarios } & 10: 00\end{array}$

$\begin{array}{ll}\text { Refrigerio } & 10: 30\end{array}$

Moderador y comentarista. Julieta Paredes

Defensora y luchadora de la humanidad: Bertha Cáceres. Universitarixs: Daniela Copa Poma,

Seny Ortuño Lucana y Alexis Josue Soria Arismendi

Máxima Acuña, la dama de la laguna azul. Universitarixs: Marina Apaza Colque, Yoli Flores Layme,

Pamela Alberto Checa y Jheanette Copa Poma

"Soy pequeña pero fuerte" Francisca “Chica” Ramírez. Universitarixs: Paola Huayta, Joacim Lima, Henry Gutiérrez, María Elena Ramos y Silvana Uscamaita

tty Cariño. Universitarixs: Yuri Escobar Bravo, Edwin Quispe y Robert Yeferson Suri $\quad 12: 10$

Ronda de preguntas y comentarios

MARTES 12 DE DICIEMBRE, AULA PRIMER AÑO

Conversando sobre "Feminismo comunitario en Bolivia." Julieta Paredes 11:00

Cierre del conversatorio

Lugar: Auditorio Taller de Historia Oral Andina (THOA)

10:40

$11: 10$

12:40

$12: 30$

Calle León Loza N 1199 esq. Asencio Padilla (Parada minibús 361), Alto San Pedro 


\section{Referencias}

Asamblea Legislativa Plurinacional de Bolivia. (2013). Ley Integral para Garantizar a las Mujeres una Vida Libre de Violencia $N^{\circ} 348$. La Paz, LP.

Belausteguigoitia, M., \& Mingo, A. (1999). Ritmos y contrapuntos, superposición de campo de los estudios de género y la educación. México, MX: Paidós.

Botella, L. (1991). Estrategias para trabajar con chicos. Londres, LDN: Open University Press.

Cabnal, L. (2010). Feminismos diversos: el feminismo comunitario. España, ES: Asociación para la Cooperación con el Sur.

Feministas Comunitarias de Abya Yala, Bolivia. (2016). El desafío de la despatriarcalización entramado para la liberación de los pueblos. La Paz, LP.

Galindo, M. (2015). La revolución feminista se llama Despatriarcalización. En Descolonización y despatriarcalización de y desde los feminismos de Abya Yala (pp. 27-50). España: ES: Asociación para la Cooperación con el Sur.

(2010). Nuestro feminismo ni maquilla, ni rellena. En: Delgado, Y., \& González, M.C. (Coords.). Mujeres en el Mundo: Multiculturalismo, violencia, trabajo, literatura y movimientos sociales (pp. 219-232). Valencia, VLC: LAINET.

Gargallo, F. (2008). El feminismo y la educación en y para nuestra América. Revista Venezolana de Estudios de la Mujer, 13(31), 17-26.

González, M. (1999). El estudio social de la ciencia en clave feminista: género y sociología del conocimiento científico. En Barral, M.J., Magallón, C., Miqueo, C., \&
Sánchez, M. D. (Eds.). Interacciones ciencia y género. Discursos y prácticas científicas de mujeres (pp. 39-62). Barcelona, BCN: Icaria, Antrazyt.

González M. C. (2010). Género y educación. Un abordaje desde las diferentes perspectivas feministas. En: Delgado, Y., \& González, M.C. (Coords.). Mujeres en el Mundo: Multiculturalismo, violencia, trabajo, literatura y movimientos sociales (pp. 321-334). Valencia, VLC: LAINET.

Heras de las, S. (2009). Una aproximación a las teorías feministas. Universitas. Revista de Filosofía, Derecho y Política, 9, 45-82.

Huanca, E. (2012). Mujeres en movilización por los derechos de los pueblos indígenas y de la madre tierra. En Fundación Tierra Marcha indígena por el TIPNIS: La lucha en defensa de los territorios (pp. 27-28). La Paz, LP: Fundación Tierra.

Iño, W. (2017). Educación y género: aproximaciones a las relaciones vigentes en la responsabilidad del cuidado. Mururata, 1(1), 7-22.

Maceira, L. (2008). El Sueño y la Práctica de Sí: Pedagogía Feminista: Una Propuesta. México DF, MXDF: El Colegio de México, Biblioteca Miguel Cosio Villegas.

Madoo, P., \& Niebrugge-Branlley, J. (1997). Teoría feminista contemporánea. En Ritzer, G. Teoría sociológica contemporánea (pp.354-407). México DF, MX: McGraw Hill.

Martínez, I., \& Ramírez, G. (2017). Despatriarcalizar y Des-colonizar la Educación. Experiencias para una Formación Feminista del Profesorado. Revista Internacional de Educación para la Justicia Social (RIEJS), 6(2), 81-95. 
DOI:

https://doi.org/10.15366/riejs2017.6.2.005.

Montenegro, C. (2016). Pedagogías feministas: posibilidades para el aprendizaje desde el partir de sí. En Actas VI Coloquio Interdisciplinario Internacional Educación, Sexualidades y Relaciones de Género y IV Congreso Género y Sociedad de pedagogías, políticas y subjetividades: recorridos y resistencias (pp.1-9). Córdoba, BCN: Universidad Nacional de Córdoba.

Ortiz, F. (1992). Historias de América: la seducción y el caos. Madrid, M: Mondadori.

Paredes, J. (2010). Hilando fino, desde el feminismo comunitario. La Paz, LP: Mujeres Creando Comunidad.

Puleo, A. (2013). Feminismo y ecología. En Manzanera, R., Miguel, C., \& Sánchez, V. (Coords.), Medio ambiente y desarrollo. Miradas feministas desde ambos hemisferios (pp. 25-42). Granada, GR: Universidad de Granada, Fundación IPADE.

(2011). Ecofemismo para otro mundo posible. Madrid, M: Catedra.

Rodríguez, M. (2002). ¿Feminización de la cultura?, Revista Debats, 76, 8-17.

i Agradezco la participación e interés que desplegaron y por el compromiso que asumieron las y los estudiantes en el aprendizaje de las teorías feministas y en la investigación que se llevó cabo durante el primer y segundo semestre. Mencionar la lista de los participantes abarcaría varios párrafos, por ello, se presenta en el anexo el programa del conversatorio que se realizó en diciembre del año 2017. También se debe destacar la participación, colaboración y contribución de los auxiliares de docencia Yessenia Valenzuela y Gonzalo Condori. Asimismo, un agradecimiento al Taller de Historia Oral (THOA) por colaborar de forma desprendida
Sánchez, M., Penna, M., \& de la Rosa, B. (2016). Somos como somos. Deconstruyendo y transformando la escuela. Madrid, M: La Catarata.

Segato, R. (2016). La guerra contra las mujeres. Madrid, M: Traficantes de Sueños.

Shiva, V. (1995). Abrazar la vida. Mujer, ecología y desarrollo. Madrid, M: Horas y Horas.

Shiva, V., \& Mies, M. (1997). Ecofeminismo. Teoría crítica y perspectiva. Barcelona, BCN: Icaria.

Subcentral de Pueblos Indígenas MojeñosYuracaré-Chimanes del TIPNIS. (2017). Respuesta de las mujeres indígenas del TIPNIS frente a las acusaciones que hace el presidente Evo Morales Ayma a las ONGs. Trinidad.

Svampa, M. (2015). Feminismos del Sur y ecofeminismo. Nueva Sociedad, 256, 127131.

Zabala, L. (2012). Descolonizar la descolonización desde los feminismos. En Sánchez, C. (Comp.), Mujeres en diálogo: Avanzando hacia la despatriarcalización en Bolivia (pp. 165-180). La Paz, LP: Coordinadora de la Mujer.

en el proceso de formación e investigación, en sus ambientes se construyeron estos conocimientos y saberes que se presentan.

ii El Plan de Estudios de esta carrera es del año 1997, por lo que hasta la fecha no se han actualizado las asignaturas y contenidos mínimos.

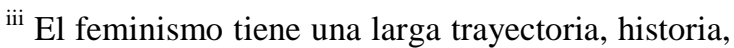
campos teóricos y epistémicos, aspectos que no se pretenden desarrollar en este artículo. 


\section{Información del Artículo / Article Information \\ Recebido em : 30/05/2018 \\ Aprovado em: 19/06/2018 \\ Publicado em: 23/12/2018 \\ Received on May 30th, 2018 \\ Accepted on June 19th, 2018 \\ Published on December 23th, 2018 \\ Contribuciones en el artículo: El autor fue responsable de la elaboración, análisis, escritura y revisión del contenido del manuscrito. Asimismo, de la aprobación de la versión final publicada.}

Author Contributions: The author was responsible for the designing, delineating, analyzing and interpreting the data, production of the manuscript, critical revision of the content and approval of the final version published.

Conflictos de interés: El autor declaró que no existe ningún conflicto de interés en el artículo.

Conflict of Interest: None reported.

\section{Orcid}

Weimar Giovanni Iño Daza

iD http://orcid.org/0000-0002-7691-4816

\section{Cómo citar este artículo / How to cite this article}

APA

Iño, W. G. D. (2018). Teorías feministas en el aula: una experiencia de formación e investigación con estudiantes de Ciencias de la Educación. Rev. Bras. Educ. Camp., 3(4), 1393-1417. DOI: http://dx.doi.org/10.20873/uft.2525$\underline{4863.2018 \mathrm{v} 3 \mathrm{n} 4 \mathrm{p} 1393}$

\section{ABNT}

IÑO, W. G. D. Teorías feministas en el aula: una experiencia de formación e investigación con estudiantes de Ciencias de la Educación. Rev. Bras. Educ. Camp., Tocantinópolis, v. 3, n. 4, set./dez., p. 1393-1417, 2018. DOI: $\quad$ http://dx.doi.org/10.20873/uft.25254863.2018v3n4p1393 